\title{
Limitations et arrêts des traitements de suppléance vitale chez l'adulte dans le contexte de l'urgence
}

\author{
Withholding and withdrawing life-sustaining therapies from adult patients in emergency care
}

\author{
A.-L. Feral-Pierssens - T. Boulain - F. Carpentier · P. Le Borgne $\cdot$ D. Del Nista $\cdot$ G. Potel $\cdot$ S. Dray \\ D. Hugenschmitt - A. Laurent - A. Ricard-Hibon - T. Vanderlinden - T. Chouihed - J. Reignier · \\ pour la Société française de médecine d'urgence et la Société de réanimation de langue française
}

Reçu le 16 mai 2018; accepté le 4 juin 2018

(C) SFMU et Lavoisier SAS 2018
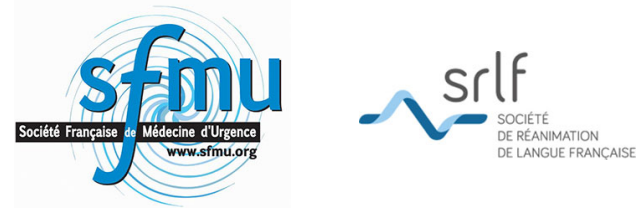

A.-L. Feral-Pierssens

Service des urgences, hôpital européen Georges-Pompidou,

20 rue Leblanc, F-75015 Paris, France

T. Boulain

Service de réanimation médicale polyvalente,

centre hospitalier régional d'Orléans, 14 Avenue de l'Hôpital,

F-45067 Orléans France

F. Carpentier

Pôle urgences médecine aiguë, hôpital universitaire des Alpes,

Avenue Maquis du Grésivaudan, F-38700 La Tronche, France

Université Grenoble Alpes, 621 avenue Centrale,

38400 Saint-Martin-d'Hères

P. Le Borgne

Service d'accueil des urgences, hôpital de Hautepierre, CHRU de Strasbourg, 1 Avenue Molière,

F-67200 Strasbourg, France

D. Del Nista

Service des urgences, centre hospitalier de Rochefort,

1 Avenue de Béligon, F-17301 Rochefort France

G. Potel

Université de Nantes, 1 Quai de Tourville,

F-44035 Nantes, France

Service des urgences, CHU de Nantes, 9 quai Moncousu,

F-44093 Nantes Cedex 1, France

S. Dray

Service de réanimation médicale, hôpital Nord,

CHU de Marseille, Chemin des Bourrely,

F-13015 Marseille, France.

D. Hugenschmitt

Samu-Smur de Lyon, hospices civils de Lyon,

3 Quai des Célestins, F-69002 Lyon, France

\begin{abstract}
A. Laurent
Université de Franche-Comté,

laboratoire de psychologie,

2 Place Saint-Jacques,

F-25000 Besançon, France
\end{abstract}

A. Ricard-Hibon

Samu-Smur 95, service d'urgences,

centre hospitalier René-Dubos,

6 Avenue de l'Île de France,

F-95300 Pontoise France

T. Vanderlinden

Service de réanimation polyvalente,

groupe hospitalier Institut catholique de Lille,

CH Saint-Philibert

115, Rue du Grand But,BP 249,

F-59462 Lomme Cedex, France

Samu-Smur, service d'urgences, hôpital Central,

CHRU de Nancy, 29 Avenue du Maréchal de Lattre de Tassigny, F-54035 Nancy France

T. Chouihed $(\bowtie)$

Samu-Smur, service d'urgences, hôpital Central,

CHRU de Nancy, 29 Avenue du Maréchal de Lattre de Tassigny,

F-54035 Nancy France

e-mail : t.chouihed@gmail.com

Inserm U1116, université de Lorraine,

9 avenue de la forêt de Haye - BP 184,

F-54500 Vandœuvre-lès-Nancy Cedex, France

J. Reignier

Médecine intensive réanimation,

CHU de Nantes, 5 Allée de l'̂̂le Gloriette,

F-44093 Nantes, France

Université de Nantes, 1 Quai de Tourville,

F-44035 Nantes, France 


\section{Introduction}

Depuis plusieurs décennies, le public, la communauté médicale, les sociétés savantes et les législateurs ont reconnu les droits des patients à décider des traitements qui leur sont administrés et, pour les patients en fin de vie, à mourir dignement. Les lois et recommandations françaises récusent l'obstination déraisonnable et imposent le recueil de la volonté du patient (via ses directives anticipées lorsqu'il est inconscient), la réalisation d'une procédure collégiale le cas échéant et l'organisation d'une prise en charge palliative incluant, si nécessaire, une sédation profonde en fin de vie [1-7].

Pour autant, ces textes n'apportent que peu d'aide aux médecins concernant les décisions de limitation et d'arrêt des traitements dans le contexte de l'urgence. Chaque année en France, 600000 personnes décèdent, dont près de $60 \%$ dans un établissement de santé. Dans $16 \%$ des cas, ces décès surviennent après un séjour bref de moins de 24 heures, souvent dans un service d'urgences. 0,1 à $0,2 \%$ des patients admis aux urgences y décèdent, constituant 5 à $7 \%$ des décès intrahospitaliers. Parmi les patients âgés institutionnalisés, un quart des décès survient au cours d'une hospitalisation [8-10].

À l'évidence, les exigences réglementaires se heurtent à la réalité du quotidien. La majorité des patients, y compris ceux souffrant de pathologies chroniques sévères, n'ont pas exprimé de souhaits précis ni rédigé de directives anticipées. Pour de nombreux patients, y compris ceux institutionnalisés dans des structures comme les établissements d'hébergement pour personnes âgées dépendantes (EHPAD), une décision de fin de vie et une prise en charge palliative n'ont pas été anticipées. Lorsque ces patients sont pris en charge en urgence, la décision de ne pas entreprendre ou d'interrompre un traitement de suppléance vitale est difficile à prendre par le médecin et son équipe. Un éventuel médecin référent du patient est rarement joignable en situation d'urgence, et la réunion de professionnels en vue d'une procédure collégiale telle que prévue par la loi pose un vrai problème pratique dans le contexte de l'urgence (y compris en préhospitalier).

Les équipes soignantes sont souvent démunies face à ces situations : les locaux des services d'urgences sont peu appropriés pour l'accueil et la prise en charge des patients en fin de vie, le temps et la disponibilité manquent en raison des flux incessants, la formation est souvent insuffisante. Les équipes médicales préhospitalières (service mobile d'urgence et de réanimation : Smur) n'ont pas pour mission première de mettre en œuvre des limitations et arrêts de traitements de suppléance qui auraient pu être anticipés et préparés antérieurement. La difficulté est de surmonter l'antagonisme apparent entre l'urgence de la prise de décision et l'élaboration complexe d'un projet thérapeutique pouvant aboutir à une limitation ou un arrêt des traitements. Ni les lois ni les dernières recommandations sur le sujet n'appor- tent de réponse spécifique à ces problématiques. C'est pourquoi la société de réanimation de langue française (SRLF) et la société française de médecine d'urgence (SFMU) ont jugé utile de réunir un groupe de travail constitué d'experts issus de la médecine d'urgence et de la réanimation dont la mission était de rédiger un texte qui se veut à la fois une prise de position sur ce sujet des limitations et arrêts des traitements de suppléance vitale dans le contexte de l'urgence, mais aussi un guide pour les professionnels exerçant dans le contexte de l'urgence [11]. Le groupe a travaillé sur la base des textes de lois, recommandations et études publiés dont une sélection est répertoriée à la suite du texte [12-43].

Ce texte sera publié dans les deux revues des sociétés savantes : Les Annales françaises de médecine d'urgence pour la SFMU et Médecine intensive réanimation pour la SRLF [11].

\section{Question 1 : Quels éléments clés sont à considérer lors de la prise de décision ?}

- En premier lieu, il est indispensable de rechercher la volonté du patient, soit par son expression directe s'il est capable de la formuler, soit au travers de ses directives anticipées ou, à défaut, au travers du témoignage de sa personne de confiance ou de ses proches [7];

- quand cette volonté n'est pas connue, l'âge ne doit pas être le seul critère pris en compte pour prendre une décision ;

- il faut tout mettre en œuvre pour récupérer le dossier médical complet du patient ainsi que les informations concernant l'autonomie antérieure, l'état cognitif et la qualité de vie préalable. Les notions d'état général très altéré, de cachexie, de perte d'autonomie, de troubles cognitifs invalidants, mais aussi les possibilités de traitements curatifs aideront à la décision du niveau de prise en charge, en particulier chez un patient souffrant d'une pathologie chronique évoluée, de pronostic vital défavorable ;

- il faut confronter le bénéfice attendu avec la lourdeur des thérapeutiques envisagées et l'impact éventuel des traitements ou d'un séjour en réanimation ;

- l'admission systématique en réanimation ne contribue pas à améliorer le pronostic et la qualité de vie des patients très âgés [24]. L'autonomie est un élément clé de la décision, et la classification des groupes iso-ressources (GIR) peut constituer un outil d'aide à la décision. Les patients GIR1 ne doivent a priori pas recevoir de traitement visant à prolonger la vie, mais uniquement ceux visant à améliorer leur confort. En dehors d'une cause simplement et très rapidement réversible, les patients déments classés GIR2 doivent être considérés comme les patients GIR1 ;

- dans les pathologies aiguës de pronostic vital et fonctionnel sombre nécessitant potentiellement un traitement d'exception, comme la craniectomie au cours des accidents vasculaires cérébraux (AVC) malins, il est licite d'engager une 
réanimation d'attente avant le recueil des informations nécessaires. Ainsi, le niveau d'engagement thérapeutique doit être discuté au cas par cas avec le patient ou à défaut ses proches et le réanimateur [34].

\section{Question 2 : Comment élaborer le projet thérapeutique en contexte d'urgence : les points clés ?}

- Chaque service doit disposer d'une procédure écrite et disponible 24 heures sur 24 exposant les principes et modalités de prise de décision et de prise en charge d'un patient en fin de vie en urgence $[6,7,29]$;

- chez le patient en capacité de décider et d'exprimer ses souhaits, il faut prendre le temps de la discussion, l'informer de sa situation et des différentes options thérapeutiques et le laisser exprimer ses souhaits ou décisions ;

- chez le patient dans l'incapacité d'exprimer ses souhaits, il faut rechercher l'existence de directives anticipées et sinon recueillir l'avis des proches [13]. Lorsqu'elles sont jugées adaptées à la situation, les directives anticipées du patient s'imposent au médecin «pour toute décision d'investigation, d'intervention ou de traitement ». Dans le contexte de l'urgence, les professionnels peuvent s'affranchir des directives anticipées si elles ne leur semblent pas adaptées ou si les proches s'opposent à leur application. Le médecin peut alors décider d'entreprendre une « réanimation d'attente » le temps d'évaluer leur pertinence ;

- dans tous les cas, il faut organiser une discussion collégiale associant médecins et paramédicaux présents [23];

- la recherche de la collégialité ne doit pas conduire à une situation d'obstination déraisonnable ;

- il faut tracer les entretiens et décisions dans le dossier médical. Il ne suffit pas de retranscrire la décision seule, mais de préciser aussi l'argumentaire dont elle est issue et les modalités d'application qui l'accompagnent ;

- la prudence s'impose devant des décisions de limitation ou d'arrêt thérapeutique antérieures. Celles-ci doivent être systématiquement réévaluées et ne peuvent être prises en compte que si elles sont jugées adaptées à la nouvelle situation et aux souhaits du patient.

\section{Question 3 : Quelle définition et quelle place pour la « réanimation d'attente »?}

- La « réanimation d'attente » est la mise en œuvre de traitements de suppléance dans un contexte d'incertitude rendant nécessaire une période d'évaluation sans faire perdre de chance au patient $[6,43]$;

- la « réanimation d'attente » peut durer quelques heures et conduire à une réévaluation (permettant de préciser le diag- nostic, l'état antérieur, une réponse aux traitements ou d'attendre l'arrivée de la famille en vue d'une annonce difficile);

- la «réanimation d'attente » peut être débutée dès la phase préhospitalière et n'implique pas une admission systématique en réanimation, elle peut être poursuivie à la salle d'accueil des urgences vitales (SAUV) [20];

- si l'incertitude se prolonge au-delà de quelques heures, la poursuite des investigations et des traitements actifs doit s'effectuer en réanimation ou en unité de surveillance continue (USC) selon le niveau de soins requis.

\section{Question 4 : Comment intégrer la possibilité de don d'organes dans le contexte de l'urgence? [42]}

- Lorsqu'un patient présente des lésions cérébrales sévères sans possibilité thérapeutique et pouvant conduire à la mort encéphalique, il doit être considéré comme un donneur potentiel quel que soit son âge ;

- une annonce anticipée de cette évolution doit être réalisée par le médecin permettant aux proches de se préparer à l'imminence du décès, de préciser les volontés émises du vivant par le patient et d'envisager un transfert médicalisé de l'éventuel donneur dans un établissement habilité aux prélèvements.

\section{Question 5 : Quelles sont les conditions de la collégialité dans le contexte de l'urgence ? $[1-4,12,28,37]$}

- Aux heures ouvrables, il faut respecter les conditions prévues par la loi. Il faut impliquer le patient, ses proches, les paramédicaux présents et un consultant extérieur adapté à la situation : le médecin traitant, un spécialiste référent, un réanimateur;

- hors heures ouvrables, une « collégialité restreinte » doit au minimum être appliquée, associant le patient, ses proches, les paramédicaux présents et, chaque fois que possible, un autre médecin, éventuellement au téléphone, dont l'avis semble le plus pertinent dans le contexte.

\section{Question 6 : Comment concilier communication avec les proches et spécificités de l'urgence?}

- Dans le contexte des décisions de limitation et d'arrêt des traitements, l'entretien avec les proches du patient est une mission difficile pour le médecin souvent en manque d'outils et craignant l'émotion qu'il va susciter. La situation est fortement asymétrique : l'un détient le savoir et exerce sa profession, l'autre est dans l'attente et vit une 
situation exceptionnelle pouvant bouleverser le cours de sa vie. Si rien ne peut modifier la difficulté du contenu à entendre, il est important d'anticiper le déroulement et le contenu de l'entretien [13,33];

- les conditions de l'entretien doivent respecter les modalités suivantes [43]:

- s'asseoir dans une pièce dédiée offrant un cadre accueillant, calme et confidentiel ;

- être en présence de l'infirmier(ière) et de l'aidesoignant(e) en charge du patient et éventuellement du (de la) psychologue du service ;

- éviter les interruptions extérieures. Si elles sont inévitables, les proches en sont informés au préalable ;

- l'entretien sera conduit de la manière suivante :

$\circ$ avoir une attitude bienveillante (empathie, disponibilité et écoute) ;

- présenter aux proches les membres présents de l'équipe ;

- informer les proches que la décision reste de la responsabilité entière de l'équipe soignante («pour prendre une décision et agir dans l'intérêt de votre proche, nous avons besoin de comprendre ce que votre proche aurait voulu que l'on fasse. Vous pouvez nous aider en témoignant sur ce sujet $»)$;

- veiller à utiliser une communication verbale et non verbale adaptées à la situation ;

- commencer par des questions sur ce que les proches savent déjà, donner les informations progressivement, laisser aux proches le temps d'intégrer l'information transmise (débit verbal adapté, arrêt dans le discours, reformulation, questionner les proches afin de savoir ce qui a été compris), comprendre et respecter leurs réactions, les aider à poser ou formuler leurs questions et à exprimer leurs émotions ;

- éviter les termes techniques ;

o conclure l'entretien et proposer du soutien aux proches ;

- l'entretien au téléphone est déconseillé, car il est vecteur d'incompréhensions et de conflits. S'il est inévitable, les principes sont ceux de l'entretien en face à face. Il convient de vérifier au préalable l'identité, le lien avec le patient et si le proche est en mesure d'avoir cette conversation importante ;

- en préhospitalier, il convient de se rapprocher au mieux de ces conditions.

\section{Question 7 : Comment organiser l'arrêt de traitements de suppléance aux urgences?}

- le contexte de l'urgence ne doit pas modifier les modalités d'arrêt des thérapeutiques ;
- devant un patient initialement pris en charge de façon invasive (intubation, amines vasoactives), l'arrêt de thérapeutiques de suppléance peut être secondairement réalisé aux urgences;

- le délai habituellement rapide de survenue du décès après un arrêt des traitements de suppléance et/ou l'instauration d'une sédation profonde incite ici à une prise en charge intégrale par l'équipe des urgences dans le lieu le plus approprié (SAUV ou unité hospitalière de courte durée (UHCD)). Cela permet d'assurer la continuité de la prise en charge du patient et de son entourage ;

- cependant, si le décès n'est pas imminent après l'arrêt des traitements de suppléance, le patient doit être admis dans un secteur d'hospitalisation, en première intention dans le service référent où il est connu ou dans une unité de soins palliatifs. Le projet doit être détaillé dans le dossier patient et transmis clairement aux équipes concernées.

\section{Question 8 : Comment instaurer une sédation profonde dans le contexte de l'urgence?}

- Il faut instaurer une sédation profonde dès que possible chez le patient en fin de vie inconscient ou présentant une souffrance réfractaire aux traitements [12];

- la sédation profonde doit associer un hypnotique et un analgésique morphinique [6,43];

- l'efficacité de la sédation et de l'analgésie doit être évaluée régulièrement et adaptée à des objectifs prédéfinis ;

- cette prise en charge doit être reportée de façon claire dans le dossier du patient ;

- le médecin veille à la compréhension de cette démarche par les proches et à la mise en œuvre de leur accompagnement.

\section{Question 9 : Quelle place pour une prise en charge palliative en urgence ?}

- La fin de vie, y compris dans le contexte de l'urgence, doit répondre aux bonnes pratiques des soins palliatifs [30];

- le secteur des urgences ne constitue pas un environnement adapté à la prise en charge optimale des situations palliatives. Par défaut, la SAUV et/ou l'UHCD sont des lieux d'accueil permettant d'instaurer provisoirement des conditions d'accueil plus adaptées ;

- tout ce qui contribue au confort du patient doit être mis en œuvre : soins de nursing adaptés, traitement de la douleur, de la dyspnée, d'un encombrement ;

- tout ce qui n'y contribue pas ou peut être source d'inconfort doit être suspendu : surveillance par scope, bilan biologique, contrôle de la glycémie capillaire ; 
- l'oxygénation est le plus souvent inutile et ne doit être utilisée que si elle améliore le confort du patient ;

- l'alimentation et l'hydratation sont à proscrire, sauf si le patient le désire. La sensation de soif est rare ; une hydratation réduite, éventuellement associée aux antisécrétoires, participe à réduire l'encombrement ;

- les professionnels des soins palliatifs peuvent être sollicités dans le contexte de l'urgence et à distance pour les retours d'expérience ou débriefing de situations difficiles ;

- il faut favoriser la formation de référents médicaux et paramédicaux à la prise en charge palliative dans le contexte de l'urgence.

\section{Question 10 : Peut-on prendre des décisions de limitations et arrêts des traitements (LAT) et les mettre en ouvre en préhospitalier ?}

- Malgré le caractère singulier de la médecine préhospitalière, les principes éthiques (bienfaisance, nonmalfaisance, autonomie et justice) doivent s'appliquer en toutes circonstances [19];

- les conditions de prise de décision précédemment décrites s'appliquent également en préhospitalier ;

- la médicalisation préhospitalière peut permettre de débuter, mais aussi d'arrêter des traitements de suppléance lorsque toutes les conditions sont réunies (informations complètes et prise en charge palliative possible) $[19,20]$;

- toutefois, en cas de difficulté, l'instauration d'une « réanimation d'attente » et un transport vers une structure hospitalière permettront de « se donner du temps » pour clarifier une situation parfois complexe ;

- l'orientation préhospitalière des patients en fin de vie ne se fait pas nécessairement vers les urgences ni même vers l'hôpital [19,21];

- l'équipe Smur peut être déclenchée pour la prise en charge d'une détresse vitale et être ensuite amenée à débuter une prise en charge palliative en lien avec la régulation médicale;

- une équipe médicalisée ne sera pas mobilisée, notamment en EHPAD, si une prise en charge palliative optimale peut être assurée rapidement sur place.

\section{Question 11 : Quels sont les intervenants participant à une décision en préhospitalier (par téléphone compris) ? [21]}

- Le patient et ses proches ;

- le médecin régulateur du Centre-15 a un rôle pivot dans cette démarche ;

- les membres de l'équipe préhospitalière ;

- le médecin traitant ou un éventuel médecin hospitalier référent, si possible ;
- les soignants d'un éventuel réseau ou d'une structure d'hébergement ;

- le réanimateur ou l'urgentiste susceptible d'accueillir le malade.

\section{Question 12 : Comment organiser une prise en charge palliative en préhospitalier ? [19,21]}

- Il est important de communiquer avec l'entourage du patient, le personnel soignant sur place et le médecin traitant ou référent afin d'obtenir leur adhésion et de ne pas exposer le patient à un inconfort ou à un transport ultérieur vers les urgences ;

- une équipe médicale préhospitalière initialement mobilisée pour assurer la prise en charge d'une détresse vitale peut, face à la constatation d'une situation de fin de vie, être amenée à débuter une sédation profonde [19,20]. Il conviendra d'organiser un relais avec les soignants éventuellement disponibles ;

- le médecin régulateur doit pouvoir, si besoin, remobiliser l'équipe préhospitalière pour une autre intervention; cela sous-entend son implication active et continue dans cette démarche. La prise en charge sera alors écourtée et complétée en intrahospitalier si nécessaire ;

- la traçabilité dans le dossier de soins et l'information des médecins correspondants sont indispensables à la continuité de la prise en charge [21].

Liens d'intérêts : les auteurs déclarent ne pas avoir de lien d'intérêt.

\section{Références}

1. Conseil de l'Europe, Assemblée parlementaire (1999) Protection des droits de l'homme et de la dignité des malades incurables et des mourants. Recommandation $\mathrm{n}^{\mathrm{o}} 1418,25$ juin 1999. http:// assembly.coe.int/nw/xml/XRef/Xref-XML2HTML-FR.asp?fileid= 16722\&lang=FR (dernier accès le 20 avril 2018)

2. République française (2002) Loi $\mathrm{n}^{\mathrm{o}} 2002-303$ relative aux droits des malades et à la qualité du système de santé du 4 mars 2002 . Journal officiel de la République française, 5 mars 2002. https:// www.legifrance.gouv.fr/affichTexte.do?cidTexte=JORFTEXT $000000227015 \&$ categorieLien $=\mathrm{id}$ (dernier accès le 20 avril 2018)

3. République française (2005) Loi $\mathrm{n}^{\mathrm{o}}$ 2005-370 du 22 avril 2005 relative aux droits des malades et à la fin de vie. Journal officiel de la République française, 23 avril 2005 https://www.legifrance. gouv.fr/affichTexte.do?cidTexte=JORFTEXT000000446240\&categorieLien $=\mathrm{id}$ (dernier accès le 20 février 2018)

4. République française (2016) Loi $n^{\circ} 2016-87$ créant de nouveaux droits en faveur des malades et des personnes en fin de vie. Journal officiel de la République française, 3 février 2016. https:// www.legifrance.gouv.fr/affichTexte.do?cidTexte=JORFTEXT $000031970253 \&$ categorieLien $=\mathrm{id}$ (dernier accès le 20 avril 2018) 
5. Société française de médecine d'urgence (2003) Éthique et urgences réflexions et recommandations. JEUR 16: 1-14

6. Société de réanimation de langue française (2010) Limitation et arrêt des traitements en réanimation adulte. Actualisation des recommandations de la SRLF. Réanimation 19: 679-698

7. Downar J, Delaney JW, Hawryluck L, Kenny L (2016) Guidelines for the withdrawal of life-sustaining measures. Intensive Care Med 42: 1003-1017

8. Rothmann C, Evrard D (2005) La mort aux urgences. JEUR 18: 3-9

9. Lalande F, Veber O (2009) La mort à l'hôpital. Rapport de l'Inspection générale des affaires sociales 2009 ; RM2009-124P: $164 \mathrm{p} \mathrm{http://www.ladocumentationfrancaise.fr/rapports-publics/}$ 104000037/index.shtml (dernier accès le 20 avril 2018)

10. Volant S (2014) 693000 résidents en établissements d'hébergement pour personnes âgées en 2011. Études et Résultats 899: 1-6

11. Feral-Pierssens AL, Boulain T, Carpentier F, et al (2018). Limitations et arrêts des traitements de suppléance vitale chez l'adulte dans le contexte de l'urgence. Médecine Intensive et Réanimation, vol. 27, in press.

12. Adams JA, Bailey DE, Anderson RA, Docherty SL (2011) Nursing roles and strategies in end-of-life decision-making in acute care: a systematic review of the literature. Nurs Res Pract 2011: 1-15

13. Azoulay E, Pochard F, Chevret S, et al (2004) Half the family members of intensive care unit patients do not want to share in the decision-making process: a study in 78 French intensive care units. Crit Care Med 32: 1832-1838

14. Baile WF, Buckman R, Lenzi R, et al (2000) SPIKES-A six-step protocol for delivering bad news: application to the patient with cancer. Oncologist 5: 302-311

15. Bioy A (2012) L'aide-mémoire de psychologie médicale et psychologie du soin. Dunod, Paris, $372 \mathrm{p}$

16. Ciais JF, Pradier C, Ciais C, et al (2007) Impact d'une équipe d'urgence spécialisée sur les hospitalisations non désirées de patients en phase terminale à domicile. Presse Med 36: 404-409

17. Couilliot MF, Vassy C, Leboul D (2011) Le temps du mourir et le temps de l'hôpital : prise en charge des patients en fin de vie aux urgences. Santé Publique 23: 269-278

18. Curtis JR, Treece PD, Nielsen EL, et al (2008) Integrating palliative and critical care: evaluation of a quality-improvement intervention. Am J Respir Crit Care Med 178: 269-275

19. Dolveck F (2010) Questions éthiques liées à la pratique de la médecine d'urgence préhospitalière au Samu et au Smur. In: Puybasset L (ed) Enjeux éthiques en réanimation. Springer, Paris, pp 447-55

20. Duchateau FX, Beaune S, Ricard-Hibon A, et al (2010) Prehospital noninvasive ventilation can help in management of patients with limitations of life-sustaining treatments. Eur J Emerg Med 17: 7-9

21. Ferrand E, Marty J; French LATASAMU group (2006) Prehospital withholding and withdrawal of life-sustaining treatments. The French LATASAMU survey. Intensive Care Med 32: 1498-1505

22. Ferrand E, Jabre P, Vincent-Genod C, et al (2008) Circumstances of death in hospitalized patients and nurses perception - French multicenter Mort-à-l'Hôpital survey. Arch Intern Med 168: 867-875

23. George NR, Kryworuchko J, Hunold KM, et al (2016) Shared decision-making to support the provision of palliative and endof-life care in the emergency department: a consensus statement and research agenda. Acad Emerg Med 23: 1394-1402

24. Guidet B, Leblanc G, Simon T, et al (2017) Effect of systematic intensive care unit triage on long-term mortality among critically ill elderly patients in France: a randomized clinical trial. JAMA 318: $1450-1459$

25. Kentish-Barnes N (2015) Communication et accompagnement en situation de fin de vie : le point de vue des familles. In: Chahraoui $\mathrm{K}$, Laurent A, Bioy A, et al (eds), Vulnérabilité psychique et clinique de l'extrême en réanimation. Dunod, Paris, pp 228-238
26. Kentish-Barnes N, Azoulay E (2016) End-of-life care in the ICU: semper ad meliora (always strive for improvement). Intensive Care Med 42: 1653-1654

27. Lakin JR, Isaacs E, Sullivan E, et al (2016) Emergency physicians' experience with advance care planning documentation in the electronic medical record: useful, needed, and elusive. J Palliat Med 19: 632-638

28. Laurent A, Bonnet M, Capellier G, et al (2017) Emotional impact of end-of-life decisions on professional relationships in the intensive care unit: an obstacle to collegiality? Crit Care Med 45: 2023-2030

29. Le Conte P, Guilbaudeau S, Batard E, et al (2005) Mise en place d'une procédure de limitation ou d'arrêt des soins actifs dans un service d'urgence. JEUR 18: 188-192

30. Le Conte P, Riochet D, Batard E, et al (2010) Death in emergency departments: a multicenter cross-sectional survey with analysis of withholding and withdrawing life support. Intensive Care Med 36: 765-772

31. McAndrew NS, Leske JS (2015) A balancing act: experiences of nurses and physicians when making end-of-life decisions in intensive care units. Clin Nurs Res 24: 357-374

32. Picard Y, Leheup BF, Piot É, et al (2016) Limitation et arrêt de traitement : collaboration entre une équipe mobile de soins palliatifs et un service de réanimation. Médecine Palliat 15: 78-85

33. Pochard F, Azoulay E, Chevret S, et al (2001) Symptoms of anxiety and depression in family members of intensive care unit patients: ethical hypothesis regarding decision-making capacity. Crit Care Med 29: 1893-1897

34. Quill TE, Holloway RG (2012) Evidence, preferences, recommendations - finding the right balance in patient care. $\mathrm{N}$ Engl J Med 366: 1653-1655

35. Reignier J, Dumont R, Katsahian S, et al (2008) Patient-related factors and circumstances surrounding decisions to forego lifesustaining treatment, including intensive care unit admission refusal. Crit Care Med 36: 2076-2083

36. Reignier J, Cottereau A, Vinatier I (2017) Limitations et arrêts des traitements ou réanimation d'attente ? https://sofia.medicalistes.fr/spip/IMG/pdf/Limitation_et_arrets_des_traitements_ou_reanimation_d_attente.pdf (dernier accès le 20 avril 2018)

37. Sedillot $\bar{N}, \bar{H}$ Holzapfel L, Jacquet-Francillon T, et al (2008) A fivestep protocol for withholding and withdrawing of life support in an emergency department: an observational study. Eur J Emerg Med 15: 145-149

38. Siddiqui S (2016) A physician's moral dilemma in the emergency department: going against a patient's perceived wishes. J Emerg Med 51: 748-749

39. Société française de médecine d'urgence (2003) $\mathrm{X}^{\mathrm{e}}$ conférence de consensus : prise en charge de la personne âgée de plus de 75 ans aux urgences, pp 1-19. http://www.sfmu.org/upload/consensus/ pa_urgs_long.pdf (dernier accès le 20 février 2018)

40. Tardy B, Venet C, Zeni F, et al (2002) Death of terminally ill patients on a stretcher in the emergency department: a French speciality? Intensive care Med 28: 1625-1628

41. Thuong M (2011) Recommandations sur l'information et l'abord des proches des donneurs potentiels d'organes et de tissus décédés après arrêt cardiaque (DDAC), dans l'optique d'un prélèvement. Ann Fr Med Urgence 1: 438-441

42. Wall J, Hiestand B, Caterino J (2015) Epidemiology of advance directives in extended care facility patients presenting to the emergency department. West J Emerg Med 16: 966-973

43. Wiese CH, Lassen CL, Bartels UE, et al (2013) International recommendations for outpatient palliative care and prehospital palliative emergencies - a prospective questionnaire-based investigation. BMC Palliat Care 12: 10 